Meta

Journal des tradlucteurs

Translators' Journal

\title{
Réflexions sur quelques termes de cinéma et de télévision
}

\section{Robert Dubuc}

Volume 13, numéro 2, juin 1968

URI : https://id.erudit.org/iderudit/002601ar

DOI : https://doi.org/10.7202/002601ar

Aller au sommaire du numéro

Éditeur(s)

Les Presses de l'Université de Montréal

ISSN

0026-0452 (imprimé)

1492-1421 (numérique)

Découvrir la revue

Citer cet article

Dubuc, R. (1968). Réflexions sur quelques termes de cinéma et de télévision. Meta, 13(2), 68-69. https://doi.org/10.7202/002601ar d'utilisation que vous pouvez consulter en ligne.

https://apropos.erudit.org/fr/usagers/politique-dutilisation/ 
irréprochable, ne touchent que des questions de détail. Nous avons relevé quelques fautes de typographie: clef à malette (molette), détenteur de pression (détendeur), train no. $22\left(\mathrm{n}^{\circ}\right)$, et quelques traits d'union superflus (papier-journal; non-disponible, non-réglable). On pourrait également mettre en doute la légitimité de certains termes donnés comme seuls équivalents (ex., superintendent: surintendant) ou placés en concurrence avec d'autres (ex., snow-plow : chasse-neige, charrue à neige; traffic manager : directeur, gérant du trafic; local : local, régional, de la place; heavy duty : dur service, grand rendement, haute capacité, grande puissance, fort débit).

À part ces quelques réserves sans grande importance, le Lexique ferroviaire présente un bon vocabulaire de vulgarisation avec lequel cheminots et employés des chemins de fer auraient avantage à se familiariser. Sachant que crate est une caisse à claire-voie, le préposé aux bagages de la gare maritime de Québec ne demandera peut-être plus à l'immigrant qui désire expédier une motocyclette à Montréal «Faut-y vous la crêter?»

Paul A. Horguelin

\section{RÉFLEXIONS SUR QUELQUES TERMES DE CINÉMA E'T DE TÉLÉVISION}

Parmi les sources où s'alimente le vocabulaire technique, l'usage n'est pas la moindre. Encore faut-il ne pas renoncer à utiliser son jugement devant les diktats de ce maître, faillible comme nous tous. L'usage souffre d'être dirigé. Dans le domaine technique, peut-être plus qu'ailleurs. Car le vocabulaire technique doit se développer rapidement au gré des circonstances, selon la fantaisie des usagers, en subissant l'influence d'autres langues et d'autres techniques.

Les droits de l'usage sont fonction de son ancienneté et de l'autorité de ceux qui le propagent. Quand un usage compte quelques lustres d'existence, c'est peine perdue que de vouloir le déraciner. De même quand les théoriciens, les critiques et les techniciens sont unanimes à employer une expression qu'on pourrait juger répréhensible, il y a fort à parier que cette "faute » deviendra "règle ".

Il est très difficile, par exemple, de déloger de la langue du cinéma et de la télévision le terme travelling ${ }^{1}$ qui a cours dans la technique cinématographique depuis au moins quarante ans ${ }^{2}$. Le mot a d'ailleurs provigné par composition: on trouve aujourd'hui travelling-avant (dolly-in), travelling-arrière (dolly-out), travelling latéral (lateral follow shot), travelling vertical (vertical follow shot). C'est un mot qu'il faut ranger au nombre des emprunts regrettables mais inévitables.

Il ne faudrait pas cependant céder ainsi devant tous les anglicismes qui contaminent la langue des studios de radiotélévision.

Pourquoi préférer back screen projection à projection par transparence qu'on peut facilement abréger en transparence? Pourquoi la désignation des plans de prise de vues se fait-elle le plus souvent en anglais en dépit d'un usage reçu en français universel depuis assez longtemps? Pourquoi les procédés de transition et

1. Prise de vue au moyen d'une caméra montée sur un chariot.

2. Cf. Jean Giraud, le Lexique trançais du cinéma des origines à 1930, Paris, Centre national de la recherche scientifique, 1958, p. 201. 
les trucages (special effects) portent-ils le plus souvent une étiquette anglaise?

On allègue pour justifier cet état de choses le caractère fonctionnel des expressions anglaises. Concédons que cette affirmation comporte une part de vérité. La langue anglaise a de ces mots courts et expressifs qui se prêtent bien à l'argot des studios.

Mais si telle était la véritable raison, préférerait-on encore l'expression anglaise à l'expression française même quand cette dernière la vaut par sa concision et sa valeur évocatrice? Les expressions accéléré (quick motion), ralenti (slow motion), marche-arrière (reverse motion), surimpression (superimpression), cache ${ }^{3}$ (mask ou matte) n'ont rien à envier à l'anglais; pourtant leur usage est loin d'être général.

La vérité tient plutôt au fait que l'on est prisonnier des termes acquis au moment de l'apprentissage du métier. Les schèmes d'expression et le découpage de la réalité sont alors inspirés de l'anglais. Faut-il dès lors s'étonner que les mots anglais s'y adaptent mieux que les français ?

Le redressement d'une terminologie dans ces conditions est quasi impossible: il exige un effort surhumain. Il est donc d'une suprême importance qu'au moment où l'on enseigne une technique, on utilise une terminologie française authentique. Il ne suffit pas pour cela de calquer les expressions anglaises. Il faut retrouver un état d'esprit, des formes d'expressions où les mots français puissent s'intégrer. On pourra alors sans crainte d'être ridicule parler d'aiguillage ${ }^{4}$ (switching), d'aiguilleur (switcher), de pupitre d'aiguillage (switching panel), de truca ${ }^{5}$ (optical printer). Ces mots respireront dans un contexte qui en facilitera l'assimilation.

On allègue que les techniciens français n'ont cure de la langue qu'ils emploient. Ils sont victimes d'un faux sentiment de sécurité. On ne se rend pas compte que les mots ne sont pas des éléments indépendants d'un code arbitraire, comme le sont les signes de l'alphabet morse. On oublie que chaque mot influe pour sa part sur l'ensemble du langage. La multiplication outrée des emprunts ne peut pas ne pas finir par influer sur la pensée. Car en un certain sens, le langage c'est la pensée même.

Il existe à travers toute la francophonie divers organismes de défense du français. C'est à eux qu'incombe de convaincre les intéressés des dangers que présente tant pour l'authenticité de la culture que pour l'originalité de la pensée l'hybridation du vocabulaire. La solution aux problèmes des terminologies ne peut tenir qu'à l'application d'un certain dirigisme qui, pour rigoureux qu'il soit, serait quand même adapté aux circonstances. Les formes que peut prendre le dirigisme peuvent être multiples. En France, par exemple, la marine de guerre règle par décret l'usage de sa terminologie. En Hongrie, l'État a fait intégrer 18000 mots dans le magyar pour le rendre apte à traduire les réalités du monde moderne. L'efficacité de telles mesures supposent une compétence incontestable chez ceux quil les appliquent, et, chez les usagers, une certaine sensibilisation à l'importance des mots.

ROBERT DUBUC

3. On dit aussi contre-cache quand le procédé est alterné.

4. Technique de commutation des images qui passent à l'antenne.

5. Tireuse optique qui permet de réaliser divers trucages cinématographiques. Cf. Lo Duca, Technique du cinéma, Paris, Presses Universitaires de France, "Que sais-je ? », 1948, p. 41. 